INPLASY

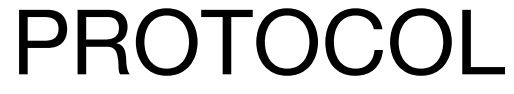

To cite: Xie et al. Traditional

Chinese medicine for

Myasthenia gravis: study

protocol for a network meta-

analysis. Inplasy protocol

202060049. doi:

10.37766/inplasy2020.6.0049

Received: 14 June 2020

Published: 14 June 2020

Corresponding author:

Rongfang Xie

vich622@163.com

Author Affiliation:

Jiangxi University of

Traditional Chinese Medicine

Support: Provincial Fund

Review Stage at time of this submission: The review has not yet started.

Conflicts of interest:

None.

\section{Traditional Chinese medicine for Myasthenia gravis: study protocol for a network meta-analysis}

\author{
Xie, R; Liu, L2; Wang, R³ Huang, C4.
}

Review question / Objective: The network meta-analysis method will be used to verify the effectiveness and safety of different types of traditional Chinese medicine in treating myasthenia gravis, and to rank the effectiveness of different treatment methods.

Condition being studied: Traditional Chinese medicine, myasthenia gravis.

Information sources: We will search the following databases: the China National Knowledge Infrastructure, Wanfang Database, Chinese Science and Technology Periodical Database, Chinese Biomedical Literature Database, Pubmed, Embase, Web of Science, and the Cochrane library. Collect all the RCT on the treatment of with Traditional Chinese medicine for Myasthenia gravis

INPLASY registration number: This protocol was registered with the International Platform of Registered Systematic Review and Meta-Analysis Protocols (INPLASY) on 14 June 2020 and was last updated on 14 June 2020 (registration number INPLASY202060049). 
Technology Periodical Database, Chinese Biomedical Literature Database, Pubmed, Embase, Web of Science, and the Cochrane library. Collect all the RCT on the treatment of myasthenia gravis with Traditional Chinese medicine.

Participant or population: Patients diagnosed with MG by internationally recognized diagnostic criteria, not restricted in age, gender, ethnicity, race and disease stage. Reluctant to accept TCM treatment, patients with severe cardiovascular diseases, mental illnesses, etc. will be excluded.

Intervention: The intervention measures of the experimental group were only Traditional Chinese medicine.

Comparator: The control group received conventional treatment of Western medicine.

Study designs to be included: Only the study of randomized controlled trial can be included.

Eligibility criteria: Only the study of randomized controlled trial can be included.

Information sources: We will search the following databases: the China National Knowledge Infrastructure, Wanfang Database, Chinese Science and Technology Periodical Database, Chinese Biomedical Literature Database, Pubmed, Embase, Web of Science, and the Cochrane library. Collect all the RCT on the treatment of with Traditional Chinese medicine for Myasthenia gravis.

Main outcome(s): The main outcome indicators include effectiveness(recognized clinical efficacy evaluation criteria), effective including basic recovery, marked effect, improvement; remission rate (no drug symptoms), relapse rate, clinical absolute score and relative score.

Additional outcome(s): Additional outcome indicators: including any related adverse reactions, The concentration of
Acetylcholine receptor antibody (AchRAb) in Serum.

Quality assessment / Risk of bias analysis: The two authors will independently assess the risk of bias (methodological quality) of the included studies based on the bias risk assessment tool recommended in the Cochrane'Risk of bias' assessment tool[21]. Including 7 items:random sequence generation, allocation concealment, blind participants and personnel, blind assessment of results, incomplete result data, selective reports and other biases. The results in each field will be divided into 3 levels: low bias risk, high bias risk and unclear bias risk. The two authors will exchange assessment results and check whether the assessment results are consistent. If there is a disagreement, the third author will participate in the discussion and determine the final result.

Strategy of data synthesis: Pairwise metaanalyses is conducted by RevMan5.3, calculated with the risk ratio (RR) and95\% confidence intervals (Cls), continuous variables will be reported as mean differences (MDs) or standardized mean differences (SMDs) with $95 \% \mathrm{Cl}$. Use WinBUGS 1.4.3 and Stata14.2 for network meta-analysis.In WinBUGS 1.4.3 software, bayesian framework is implemented by the Markov chain Monte Carlo (MCMC) method.Make evidence network diagram, correct-compare funnel diagram and conduct inconsistency test in Stata14.2 software. Simultaneously calculate the value of surface under the cumulative ranking curves(SUCRA) and the area under the SUCRA curve in order to rank the efficacy of various interventions. The value range is $0 \sim 100$. The larger the value, the larger the area under the curve indicates the intervention The greater the likelihood of being the best intervention.

Subgroup analysis: If the Chi-squared test and Higgins 12 test detect obvious heterogeneity between studies, We will conduct a subgroup analysis from the following aspects: different types of TCM, 
treatment time, MG classification, course of disease and so on.

Sensibility analysis: In order to ensure the Credibility of the research results, we will conduct a sensitivity analysis of the included literature and will eliminate lowquality literature.

Language: Chinese, English.

Country(ies) involved: China.

Keywords: Traditional Chinese medicine, myasthenia gravis, protocol, network metaanalysis.

Contributions of each author:

Author 1 - Rongfang Xie - Author 1 drafted the manuscript.

Author 2 - Liting Liu - The author provided statistical expertise.

Author 3 - Ruiqi Wang - The author contributed to the development of the selection criteria, and the risk of bias assessment strategy.

Author 4 - Chunhua Huang - The author read, provided feedback and approved the final manuscript. 